\title{
Estado nutricional, condición física, rendimiento escolar, nivel de ansiedad y hábitos de salud en estudiantes de primaria de la provincia del Bio Bío (Chile): Estudio transversal
}

\author{
Nutritional status, fitness, school performance, anxiety level \\ and health habits in primary school in the Bio Bío province of \\ Chile: A cross-sectional study
}

\begin{abstract}
In recent years, obesity has increased and its presence has been associated with poor physical condition, psychological problems and poor school performance. The aim of this study was to describe nutritional status, physical condition, school performance, anxiety level, and health habits in primary schoolchildren (6-7 years old). We also determined differences among overweight/obese and normal weight groups. Two studies were conducted among 1st and 2 nd graders $(n=276)$. In both studies we measured: anthropometry, physical fitness, and academic performance. In addition, in the first study, waist circumference was measured and, in the 2nd study, health habits and anxiety symptoms were obtained. Boys and girls had high levels of overweight and obesity. Those who had higher levels of BMI/age had worse physical fitness, spent less hours per week in scheduled physical activity, ate more unhealthy foods. There were no differences in academic performance. Overweight/obese girls had lower anxiety symptoms than normal weight girls. While these results shed light on current nutritional status, physical, academic and mental condition of a sample of primary school children, further studies are needed to confirm the elevated levels of obesity and overweight detected.

Key words: nutritional status, primary education, school performance, healthy behaviors, physical fitness, mental health
\end{abstract}

\section{INTRODUCCIÓN}

En las últimas décadas, Chile ha sufrido un gran cambio demográfico y epidemiológico, que ha provocado un envejecimiento de la población y profundos cambios en su perfil nutricional. En cuanto a los hábitos de alimentación y de actividad física (AF), las familias han reemplazado el consumo de comidas caseras por el de comidas rápidas y de alimentos con alta concentración calórica, grasas saturadas y azúcares (especialmente bebidas gaseosas)' Este tipo de alimentación queda muy lejos de las dietas
Igor Cigarroa ${ }^{1,2}$, Carla Sarqui ${ }^{1}$, Danila Palma ${ }^{1}$, Nicole Figueroa ${ }^{3}$, María Castillo ${ }^{4}$, Rafael Zapata-Lamana ${ }^{3}$, Rosa Escorihuela

Este trabajo fue recibido el 5 de noviembre de 2016. Aceptado con modificaciones: 22 de mayo de 2017. Aceptado para ser publicado: 07 de julio de 2017.

saludables recomendadas (Ministerio de Salud de Chile (Minsal)) que incluyen diariamente 5 raciones de verduras y frutas de colores distintos, legumbres y pescados al menos 2 veces por semana ${ }^{2}$.

Según los resultados de la Encuesta Nacional de consumo alimentario el $95 \%$ de la población chilena no tiene una alimentación saludable 3 . Además, cerca del $80 \%$ de la población no realiza AF regularmente (proporción mayor en mujeres) y los niños solo realizan la actividad asociada a la materia de educación física en la escuela ${ }^{4}$, 
lo cual queda muy alejado del tiempo mínimo de 60 minutos diarios de AF recomendado por la Organización Mundial de la Salud (OMS) para niños de 5-17 años 5 . Con todo esto, tenemos una población sedentaria, que ha aumentado la prevalencia de malnutrición por exceso (sobrepeso y obesidad) y las enfermedades crónicas no transmisibles (ECNTs). En la actualidad, Chile es el $6^{\circ}$ país miembro con mayor malnutrición por exceso en menores de 6 años $(34,4 \% \text { hombres y 33,7\% mujeres })^{6}$. A partir de esta edad, la prevalencia de malnutrición por exceso sufre un alza importante, llegando en escolares de $1^{\circ}$ año básico a un $53,7 \%$ en niñas y un $53 \%$ en niños. Esta prevalencia es relativamente similar por género y aumenta ligeramente en la zona sur del país ${ }^{7}$. La obesidad escolar está asociada a la obesidad adulta. Los niños y niñas tienen 5 y 9 , respectivamente veces más posibilidades de convertirse en adultos obesos que los niños y niñas con normopeso ${ }^{8}$. Las principales consecuencias de un escolar con malnutrición por exceso son la presencia de factores de riesgo cardiovascular, intolerancia a la glucosa y resistencia a la insulina9. La obesidad también se asocia a hipo-actividad, condición física baja, problemas psicológicos incluyendo sintomatología depresiva o ansiedad ${ }^{10}$. Estudios previos han examinado la relación entre obesidad infantil y rendimiento académico (puntajes en test de matemáticas y lenguaje). Datar et al (2004) en una muestra representativa de más de 11.000 niños y niñas estadounidenses (4-5 años) detectaron que los niños con sobrepeso tenían puntajes más bajos en los test estandarizados de matemática y lenguaje que los niños con peso normal ${ }^{11}$. Resultados similares comienzan a aparecer en muestras representativas de escolares chilenos al estudiar la relación entre la malnutrición por exceso, hábitos de salud y el rendimiento académico medido con test estandarizados ${ }^{12}$. Sin embargo, son menos los estudios que analizan la relación entre el estado nutricional, la salud mental en edades infantiles. Al día de hoy, según la bibliografía revisada, no tenemos conocimiento de ningún estudio previo en Chile en donde se analicen estas relaciones en estudiantes de $1^{\circ}$ y $2^{\circ}$ año de enseñanza básica. Sobre la base de lo expuesto se aportan dos estudios. El primero tiene como objetivo analizar y asociar el estado nutricional, el rendimiento escolar y la condición física en niños y niñas con normopeso y con malnutrición por exceso de $1^{\circ}$ y $2^{\circ}$ año básico y el segundo tiene como objetivo establecer la relación de los parámetros nutricionales con variables de rendimiento escolar y condición física y, además, evaluar variables de salud mental, hábitos de ingesta alimentaria y actividad física.

\section{MATERIALES Y MÉTODO $1^{\circ}$ Estudio}

Participaron 478 escolares (248 niños y 230 niñas) de 4-6 años de $1^{\circ}$ y $2^{\circ}$ año básico de tres centros educativos públicos de una comuna urbana de la provincia del Bio Bío (Chile) fueron autorizados por sus tutores para participar en el estudio. Los criterios de inclusión fueron: estudiantes con peso normal, sobrepeso u obesidad (no bajo peso u obesos mórbidos). Los criterios de exclusión fueron: estudiantes con patologías neurológicas, osteo-articulares, cardiorespiratorias, metabólicas o tumorales que pudieran interferir en la evaluación de la condición física o que alteraran su peso, apetito o rendimiento académico e inasistencia a las evaluaciones. Se excluyeron 260 escolares. Así, la muestra se constituyó por 218 (114 niños y 104 niñas) (Tabla I). En ambos estudios los padres de los participantes informaron la edad y el lugar de procedencia. La distribución según sexo fue similar en ambos estudios a valores de encuestas nacionales ( $51 \%$ niños y $49 \%$ niñas), no así el área de residencia (13\% área rural y $87 \%$ área urbana) encontrando un mayor porcentaje de niños con residencia rural $(22,9 \%$ en el $1^{\circ}$ estudio y un $15,5 \%$ en el $2^{\circ}$ ).

\section{Evaluación antropométrica}

El peso se obtuvo con una balanza (SECA ${ }^{\circledR}$, Modelo 713 , USA) de una precisión de $0,2 \mathrm{~kg}$ y la talla con un cartabón de pared de una precisión de $0,1 \mathrm{~cm}$. La obesidad abdominal se midió a través del perímetro de cintura (PC) con una cinta métrica antropométrica marca Lufkin W606PM de una precisión de $0,1 \mathrm{~cm}$ colocada a la altura de la cresta ilíaca. El índice de masa corporal se corrigió por los estadios de maduración de Tanner preguntado a la madre de los menores. El ((IMC)/edad), la talla/edad y el PC/edad se calcularon según las curvas del Center for Disease Control and Prevention (CDC/NCHS) utilizadas en Chile para la evaluación nutricional de niños de 0-9 años ${ }^{13}$.

\section{Evaluación de la condición física}

Se utilizó el test de sentarse y pararse. Es una de las pruebas más usadas en baterías de tests nacionales e internacionales en niños y adolescentes para medir fuerza muscular de miembros inferiores ${ }^{14,15}$. En Chile, se utiliza en el Programa vida sana para medir la resistencia muscular. El estudiante debía sentarse y pararse en una silla, sin la ayuda de los brazos, el mayor número de veces que pueda durante $30 \mathrm{~s}$. Las evaluaciones se hicieron en una sala del centro educativo.

\section{Evaluación del rendimiento escolar}

Se midió tomando las notas promedio del $1^{\circ}$ semestre del curso actual obtenidas por los niños (as) en las asignaturas de matemáticas y lenguaje. Los promedios van de 1,0 a 7,0 , donde 4,0 es la nota aprobatoria. No se consideraron diferencias de exigencia académica, ya que todos loscentros educativos eran públicos y por ende están sujetos a las mismas bases curriculares y programas de estudio dados por el ministerio de educación (MINEDUC).

\section{$2^{\circ}$ Estudio}

Participaron 72 escolares (43 niños y 29 niñas) de entre 6-7 años de $1^{\circ}$ año básico de 2 centros educativos públicos de la misma comuna que el estudio 1 . Se usaron los mismos criterios de inclusión y exclusión que el estudio 
1 excluyendo a 14 escolares, participando finalmente 58 escolares (37 hombres y 21 mujeres) (Tabla I).

Evaluación antropométrica y de rendimiento escolar: Evaluación de la condición física: 1) Salto horizontal a pies juntos: Fue medido desde una línea horizontal, se pidió al escolar que saltase lo más lejos que pudiera, a pies juntos y sin impulso previo. Se midió la distancia máxima alcanzada y se expresó en centímetros $(\mathrm{cm})$. Esta prueba es una de las más utilizadas y se considera un índice general de fuerza muscular ${ }^{16}$. Además, también se utiliza en el programa Vida Sana del Minsal (Chile) para medir la condición física en la población entre 2-5 años ${ }^{15}$. 2) Carrera de 12 metros: Se midió el tiempo, expresado en segundos, que tardaba el escolar en recorrer una distancia de 12 metros $(m)^{17}$. Los niños se familiarizaron con las pruebas una semana antes de la evaluación, cada prueba se realizó 3 veces y luego se calculó la media.

Cuestionario de hábitos de ingesta: Evalúa la calidad de hábitos de ingesta en niños y adolescentes en categorías alimentarias (Desayuno/once, almuerzo/ cena, colación y snack). Los alimentos ricos en grasa y azúcar se ponderaron como no saludables, los alimentos normograsos elaborados, se ponderaron como regulares y los alimentos ricos en fibra y bajos en grasas saturadas y/o azucares se ponderaron como saludables. Se contabilizó el consumo de comidas no saludables ${ }^{1}$.

Cuestionario de hábitos de actividad de física (HAF): Cuestionario creado por el Instituto de nutrición y tecnología de los alimentos de Chile (INTA). Se utilizó para cuantificar los hábitos de AF de un niño o adolescente durante la semana, incluyendo una variedad de actividades que van de hábitos sedentarios hasta AF sistemática. Se utilizó la categoría de AF sistemática semanal (clases de educación física y deportes), divididas en menos de 2 horas, entre 2 y 4 horas y más de 4 horas $^{18}$.

Escala de ansiedad infantil de Spence (SCAS): Está formada por 38 ítems y evalúa los trastornos de ansiedad infanto-juvenil más frecuentes, dividiéndolos en 6 subescalas. Se responde con una escala de 4 opciones tipo Likert, que va desde 0 (nunca) a 3 (siempre). Se usó la SCAS versión española ${ }^{18}$. Se califica mediante la suma de los puntos obtenidos en cada ítem. A mayor puntaje, más ansiedad. Se escogió esta prueba por su consistencia interna ${ }^{19}$. Además, la SCAS ha mostrado que es capaz de distinguir entre muestras con trastornos de ansiedad y muestras no clínicas $^{20}$. Los padres o tutores de los niños (as) rellenaron los cuestionarios y la escala de

\section{Tabla 1}

Valores descriptivos de las variables antropométricas y el estado nutricional de los estudios 1 y 2.

\begin{tabular}{|c|c|c|c|c|c|c|}
\hline & \multicolumn{2}{|c|}{ Estudio 1 (n=218) } & \multicolumn{4}{|c|}{ Estudio $2(n=58)$} \\
\hline & $\hat{~}(\mathbf{n}=114)$ & ㅇ $(\mathbf{n}=\mathbf{1 0 4})$ & p valor & $\hat{~}(\mathbf{n}=\mathbf{3 7})$ & $q(\mathbf{n}=\mathbf{2 1})$ & p valor \\
\hline \multicolumn{7}{|l|}{ Variables antropométricas (media \pm ETM) } \\
\hline Edad (años) & $6,5 \pm 0,58$ & $6,6 \pm 0,57$ & NS & $6,1 \pm 0,09$ & $5,9 \pm 0,07$ & NS \\
\hline Peso (kg) & $27,95 \pm 0,56$ & $27,66 \pm 0,56$ & NS & $26,60 \pm 1,03$ & $26,63 \pm 1,29$ & NS \\
\hline Talla (m) \pm & $1,22 \pm 0,01$ & $1,22 \pm 0,01$ & NS & $1,22 \pm 0,01$ & $1,21 \pm 0,01$ & NS \\
\hline Talla/edad (m/años) & $0,189 \pm 0,002$ & $0,186 \pm 0,001$ & NS & $0,20 \pm 0,00$ & $0,20 \pm 0,00$ & NS \\
\hline $\mathrm{IMC}\left(\mathrm{kg} / \mathrm{m}^{2}\right)$ & $18,70 \pm 0,29$ & $18,46 \pm 0,26$ & NS & $17,76 \pm 0,53$ & $18,14 \pm 0,64$ & NS \\
\hline Perímetro de cintura $(\mathrm{cm})$ & $58,46 \pm 0,68$ & $57,18 \pm 0,61$ & NS & ------- & ------- & \\
\hline Estado nutricional (distribución*) & \multirow{2}{*}{\multicolumn{3}{|c|}{ Chi-cuadrado }} & & & \\
\hline Talla/edad (m/años) & & & & & & \\
\hline Talla alta (>p95) \% (n) & $6,1 \%(7)$ & $5,8 \%(6)$ & NS & $13,5 \%(5)$ & $14,3 \%(3)$ & NS \\
\hline Talla normal (p5 - p95) \% (n) & $93,9 \%(107)$ & $93,3 \%(97)$ & NS & $86,5 \%(32)$ & $85,7 \%(18)$ & \\
\hline Talla baja $(<\mathrm{p} 5) \%$ (n) & $0,0 \%(0)$ & $1,0 \%(1)$ & & $0,0 \%(0)$ & $0,0 \%(0)$ & \\
\hline \multicolumn{7}{|l|}{$\mathrm{IMC}\left(\mathrm{kg} / \mathrm{m}^{2}\right)$} \\
\hline Obesidad (p>95) \% (n) & $36,0 \%(41)$ & $33,7 \%(35)$ & NS & $32,4 \%(12)$ & $38,1 \%(8)$ & NS \\
\hline Sobrepeso (p85 - p95) \% (n) & $23,7 \%(27)$ & $25,0 \%(26)$ & & $10,8 \%(4)$ & $23,8 \%(5)$ & \\
\hline Peso normal (p10-p85) \% (n) & $39,5 \%(45)$ & $41,3 \%(43)$ & & $56,8 \%(21)$ & $38,1 \%(8)$ & \\
\hline Bajo peso $(<\mathrm{p} 10) \%(\mathrm{n})$ & $0,9 \%(1)$ & $0,0 \%(0)$ & & $0,0 \%(0)$ & $0,0 \%(0)$ & \\
\hline \multicolumn{7}{|l|}{ Obesidad abdominal $(\mathrm{cm})$} \\
\hline Perímetro de cintura (>p90) \% (n) & $14 \%(16)$ & $9,6 \%(10)$ & NS & ------- & -------- & \\
\hline Perímetro de cintura $(<\mathrm{p} 90) \%(\mathrm{n})$ & $86 \%(98)$ & $90,4 \%(94)$ & NS & ------- & -------- & \\
\hline
\end{tabular}


ansiedad. Las evaluaciones se hicieron en las mismas condiciones ambientales que el estudio 1.

\section{Análisis estadístico}

La muestra se dividió por cursos (en el estudio 1) y se generaron 4 grupos por estado nutricional y género: niños-normopeso ( $h-n p)$, niños-sobrepeso/obesidad ( $h$-spo), niñas-normopeso (m-np), niñas-sobrepeso/obesidad (m-spo). Las variables se expresaron en media \pm error estándar de la media y los indicadores del estado nutricional en porcentaje. Los datos se analizaron utilizando el programa SPSS (v.19.0, SPSS Inc., USA). La distribución normal de los datos se midió con el test de K-S. Para comparaciones de variables paramétricas entre dos grupos se utilizó la prueba t de student y cuando se compararon más de dos grupos se utilizó un anova de dos vías y post hoc de Duncan. Para la comparación entre variables categóricas fue utilizado el test chi-cuadrado. En el caso de las variables no paramétricas, se utilizó la prueba $U$ de Mann-Whitney en la comparación de dos grupos y la prueba $\mathrm{H}$ de Kruskal-Walis para comparar más de dos grupos. Para la asociación entre las variables se utilizó la correlación de Pearson o Spearman. El nivel de significancia empleado fue de $p \leq 0,05$.

\section{Comité de ética}

El $1^{\circ}$ y $2^{\circ}$ estudio fueron aprobados por el comité de ética de la Universidad Santo Tomás, Los Ángeles (Chile) y ambos se realizaron previa firma de cartas de consentimiento. Todos los procedimientos siguieron los principios éticos para las investigaciones médicas en seres humanos según la declaración de Helsinki actualizada en la $64^{\mathrm{a}}$ Asamblea General, Fortaleza, Brasil (2013).

\section{RESULTADOS \\ $1^{\circ}$ Estudio}

Los niños (6,5 años) y niñas (6,6 años) tenían un IMC/edad que se encontraban en el rango de obesidad y sobrepeso respectivamente. El 59,2\% presentó malnutrición por exceso, de los cuales, $24,3 \%$ tenía sobrepeso $(23,7 \%$ niños y $25,0 \%$ niñas) y el $34,9 \%$ eran obesos ( $36,0 \%$ niños y $33,7 \%$ niñas). En relación con la talla/edad los niños y niñas se encontraban en el rango de talla normal para su edad. Solo el $1 \%$ de las niñas presentó talla baja con respecto a la edad. De acuerdo con el perímetro de cintura/edad ambos sexos presentaron una media menor al p90 $(64,4)$, lo que indicó bajo riesgo cardiovascular en la adultez. Pese a esto, un $14 \%$ de niños y un 9,6\% de niñas mostraron un PC mayor a p90 (Tabla 1). El análisis de la varianza mostró efectos significativos del estado nutricional en $1^{\circ}$ año básico en el PC. Niños y niñas normopeso mostraron significativamente menos PC que los grupos sobrepeso/obesidad. El grupo h-spo estuvo muy cerca del p90. En relación con la condición física, el grupo h-spo realizó menos cantidad de repeticiones en el test de sentarse y pararse que todos los otros grupos. Con respecto al rendimiento académico, no se observaron diferencias entre los grupos (Tabla 2). En el $2^{\circ}$ año básico, se observó el mismo efecto del estado nutricional sobre el PC que en el $1^{\circ}$ año básico. Los escolares normopeso de ambos sexos presentaron menos PC que los grupos sobrepeso/obesidad. En relación con el test sentarse pararse, los grupos h-spo y m-spo tuvieron un peor rendimiento comparado con los niños y niñas con normopeso. En relación a las variables de rendimiento académico, no se observaron diferencias dadas por género o por estado nutricional (Tabla 2).

El análisis de correlaciones reveló que independiente del género, el bajo rendimiento en el test de sentarse pararse se asoció con un valor alto de IMC $(r=-0,264, p=0,005$ niños y $r=-0,436, p=0,000$ en niñas $)$ y de $P C(r=-0,272, p=$ 0,004 niños y $r=-0,496, p=0,000$ niñas). Sólo en las niñas se observó una asociación débil entre el bajo rendimiento en el test de condición física y un bajo rendimiento académico en matemáticas $(r=0,197, p=0,046)$.

\section{$2^{\circ}$ Estudio}

Se confirmaron los resultados encontrados en el estudio 1. Por un lado, al separar por género, se observó que los niños (6,1 años) y las niñas (5,9 años) tenían un IMC/edad de sobrepeso. El 41,7\% de los escolares presentó malnutrición por exceso, de los cuales $15,5 \%$ tenían sobrepeso $(10,8 \%$ en niños y $23,8 \%$ en niñas) y $36,2 \%$ obesidad ( $32,4 \%$ niños y $38,1 \%$ niñas). No se encontraron diferencias en las variables nutricionales por género. En relación a la talla, ambos géneros presentaron una altura correspondiente a la talla normal en relación a la edad (1,22 hombres y 1,21 mujeres), ningún escolar presentó talla baja. El análisis de la varianza mostró efectos significativos del género y del estado nutricional en el test de condición física salto horizontal a pies juntos. Los escolares con sobrepeso/obesidad, principalmente las niñas, saltaron distancias más cortas que los escolares con normopeso. No se encontraron diferencias de género o del estado nutricional en el rendimiento académico y en los niveles de ansiedad. Cuando se hizo un análisis más detallado, se observó que los grupos normopeso tuvieron una tendencia a presentar mayores puntajes en el test de ansiedad que los grupos con sobrepeso/obesidad (significativo en la categoría ataques de pánico y agorafobia y trastorno de ansiedad y separación y puntaje total). En particular, las niñas del grupo $n-n p$ presentaron un puntaje total mayor que las niñas del grupo $n$-spo $(p=0,065)$. El consumo de alimentos no saludables fue significativamente mayor en los niños con sobrepeso/obesidad en la categoría almuerzo/cena y en las niñas con sobrepeso/obesidad en la categoría snack con relación a los grupos normopeso. Ningún grupo realizó más de 4 horas semanales de AF sistemática, aunque un mayor porcentaje de escolares normopeso de ambos sexos declaró realizar entre 2 y 4 horas semanales de AF sistemática (Tabla 3). El análisis de correlación mostró que, en las niñas los altos puntajes en la escala de ansiedad infantil (SCAS) (mayor ansiedad) se asociaron con bajos puntajes en matemáticas $(r=-0,633$, $p=0,004)$ y lenguaje $(r=-0,488, p=0,034)$. 
Tabla 2

Comparación de grupos dada por género y estado nutricional del estudio 1

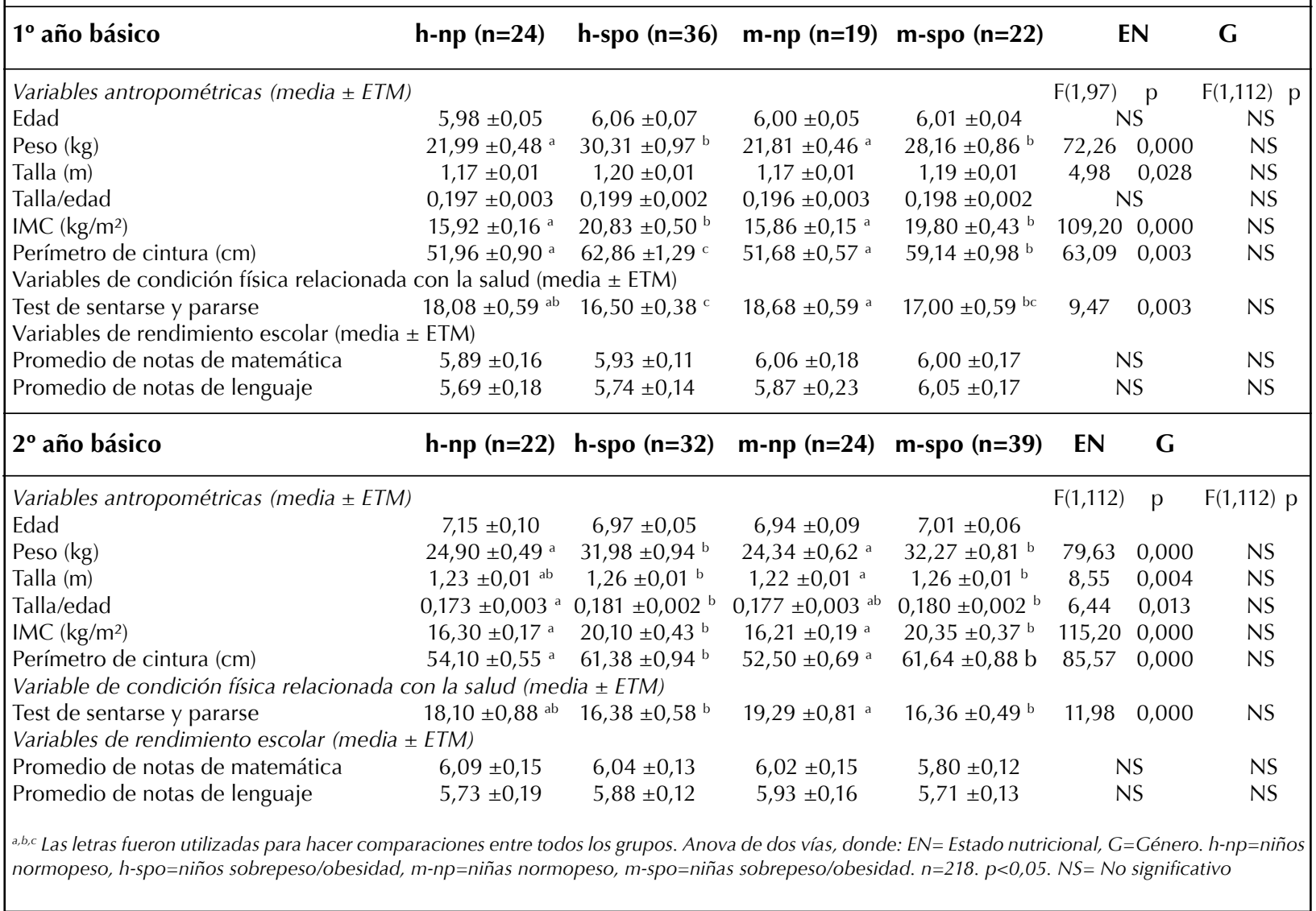

\section{DISCUSIÓN}

Los porcentajes de malnutrición por exceso sobrepasaron en niños y niñas en el estudio 1 y en niñas en el estudio 2 a los porcentajes de normopeso (Tabla 1). En ambos estudios, y para ambos sexos, la prevalencia de obesidad fue mayor que la de sobrepeso, superando la prevalencia nacional $(28,3 \%$ en hombres y $22,3 \%$ en mujeres). Valores similares de malnutrición por exceso ya habían sido descritos en estudios chilenos en población con edad y zona de residencia similar (27,0\% sobrepeso y $39,9 \%$ obesidad $)^{21}$ y son considerablemente más altos a los porcentajes descritos en Argentina (17,9\% sobrepeso y $16,7 \%$ obesidad $)^{22}$ y Bolivia $(29,6 \% \text { sobrepeso y } 4,9 \% \text { obesidad })^{23}$. Los datos no revelaron diferencias de IMC/edad entre género, en concordancia con las estadísticas nacionales ${ }^{3,4}$. En relación con la talla/ edad, los resultados indicaron un bajo porcentaje de niños y niñas con baja talla respecto a valores de referencia nacional (2,4\% hombres y $2,9 \%$ mujeres). El marcado incremento de la obesidad y sobrepeso en los 5 y 6 años podría asociarse a lo que se conoce como el "rebote de adiposidad". Este fenómeno ese refiere al aumento normal del IMC, después que el niño(a) ha alcanzado el menor punto de adiposidad, normalmente a los 6-7 años ${ }^{24}$, asociándose a obesidad y síndrome metabólico en la adultez cuando se da precozmente ${ }^{25}$. Otros factores podrían explicar en parte los altos porcentajes de malnutrición por exceso encontrados en nuestra muestra. Por un lado, se ha observado que en áreas urbanas hay un menor porcentaje de malnutrición por exceso en comparación con áreas rurales, considerándose el área urbana un factor protector. Por otro lado, las encuestas nacionales indican que hay mayor prevalencia de obesidad en la zona sur del país, principalmente debido al clima frío y húmedo que propicia consumo de alimentos con mayor densidad calórica y a permanecer más tiempo dentro de las casas. El estudio se hizo en 2 ciudades del sur del país (VIII región), con un porcentaje de residencia rural mayor a la media nacional ${ }^{26}$.

La condición física, en particular la capacidad aeróbica y la fuerza muscular se consideran importantes marcadores relacionados con la salud en la infancia ${ }^{27}$. En el presente estudio hemos abordado la relación entre el estado nutricional medido por IMC y la condición física medida a través de tests de campo, basándonos en los test utilizados en el programa del Gobierno de Chile "Vida 
Tabla 3

Comparación de grupos dada por género y estado nutricional del estudio 2

\begin{tabular}{|c|c|c|c|c|c|c|c|}
\hline & h-np $(n=21)$ & h-spo $(n=16)$ & $m-n p \quad(n=8)$ & m-spo $(n=13)$ & & EN & G \\
\hline \multicolumn{5}{|l|}{ Variables antropométricas (media \pm ETM) } & $F(1,54)$ & $\mathrm{p}$ & $F(1,54) \quad p$ \\
\hline Edad (años) & $6,0 \pm 0,07$ & $6,1 \pm 0,18$ & $6,0 \pm 0,00$ & $5,85 \pm 0,10$ & \multicolumn{2}{|c|}{ NS } & NS \\
\hline Talla (m) & $1,20 \pm 0,01^{\mathrm{ab}}$ & $1,24 \pm 0,02^{b}$ & $1,18 \pm 0,01^{\mathrm{a}}$ & $1,22 \pm 0,01^{\mathrm{b}}$ & 8,74 & 0,005 & NS \\
\hline Talla/edad (m/años) & $0,201 \pm 0,003^{\mathrm{ab}}$ & $0,204 \pm 0,005^{\mathrm{ab}}$ & $0,197 \pm 0,001^{\mathrm{a}}$ & $0,210 \pm 0,004^{b}$ & 4,39 & 0,041 & NS \\
\hline $\mathrm{IMC}\left(\mathrm{kg} / \mathrm{m}^{2}\right)$ & $15,5 \pm 0,19^{a}$ & $20,8 \pm 0,64^{b}$ & $15,3 \pm 0,37^{a}$ & $19,9 \pm 0,62^{\mathrm{b}}$ & 94,20 & 0,000 & NS \\
\hline Carrera de 12 metros (s) & $2,98 \pm, 04$ & $3,10 \pm 0,07$ & $3,13 \pm 0,09$ & $3,20 \pm 0,09$ & \multicolumn{2}{|c|}{ NS } & NS \\
\hline \multicolumn{8}{|c|}{ Variables de rendimiento académico (media \pm ETM) } \\
\hline Promedio de notas de matemática & $6,01 \pm 0,17$ & $5,59 \pm 0,20$ & $5,99 \pm 0,33$ & $5,84 \pm 0,25$ & \multicolumn{2}{|c|}{ NS } & NS \\
\hline $\begin{array}{l}\text { Promedio de notas de lenguaje } \\
\text { Variable de salud mental (media } \pm \text { ETM) }\end{array}$ & $5,75 \pm 0,20$ & $5,19 \pm 0,27$ & $5,59 \pm 0,38$ & $5,45 \pm 0,34$ & \multicolumn{2}{|c|}{ NS } & NS \\
\hline Miedo al daño físico & $5,24 \pm 0,91$ & $5,56 \pm 0,86$ & $5,50 \pm, 1,17$ & $5,46 \pm 0,55$ & \multicolumn{2}{|c|}{ NS } & NS \\
\hline Trastorno obsesivo compulsivo & $4,48 \pm 0,91$ & $2,94 \pm 0,64$ & $5,12 \pm, 1,25$ & $2,69 \pm 0,82$ & 4,28 & 0,043 & NS \\
\hline Trastorno de ansiedad generalizada & $6,48 \pm 0,87$ & $5,94 \pm 0,93$ & $7,5 \pm 1,36$ & $4,62 \pm 0,73$ & \multicolumn{2}{|c|}{ NS } & NS \\
\hline \multirow{2}{*}{\multicolumn{5}{|c|}{$\begin{array}{l}\text { Variables de hábitos de salud (distribución) } \\
\text { Consumo de comidas no saludables ( } \uparrow \text { en grasa y azúcar) }\end{array}$}} & \multicolumn{3}{|c|}{ Chi-cuadrado } \\
\hline & & & & & $\partial(n=$ & $=37)$ & of $(\mathrm{n}=21)$ \\
\hline Desayuno & $85,7 \%(18)$ & $100 \%(16)$ & $75,5 \%(5)$ & $100 \%(13)$ & 0,1 & 115 & 0,058 \\
\hline Almuerzo/cena & $66,7 \%(14)$ & $93,8 \%(15)$ & $50 \%(4)$ & $76,9 \%(10)$ & 0,0 & 947 & 0,204 \\
\hline Colación & $85,7 \%(18)$ & $100 \%(16)$ & $62,5 \%(5)$ & $92,3 \%(12)$ & 0,1 & 115 & 0,091 \\
\hline Snack & $81,0 \%(17)$ & $93,8 \%(15)$ & $50 \%(4)$ & $100 \%(13)$ & 0,2 & 259 & 0,005 \\
\hline \multicolumn{8}{|c|}{ Actividad física sistemática (clases de educación física y deportes) } \\
\hline 2 a 4 horas semanales $\%(n)$ & $71,4 \%(15)$ & $37,5 \%(6)$ & $87,5 \%(7)$ & $30,8 \%(4)$ & 0,0 & 039 & 0,11 \\
\hline
\end{tabular}

Sana"15. En la prueba de salto longitudinal se observaron diferencias entre género y entre estado nutricional. Las niñas con sobrepeso/obesidad saltaron distancias más cortas que los otros grupos, en concordancia con otros estudios en niños y adolescentes ${ }^{28,29}$. En esta línea, CastroPiñero et al (2009) compararon el rendimiento en diferentes pruebas de fuerza muscular (salto longitudinal y vertical) en escolares de 6-17años $(n=2778)$ encontrando que los grupos normopeso presentaron un mejor rendimiento que los grupos con sobrepeso/obesidad ${ }^{29}$.

En nuestro estudio, los grupos con mayor IMC también presentaron peor rendimiento en el test sentarse pararse en comparación con los grupos normopeso, principalmente en las niñas que hicieron como media un $14 \%$ menos de repeticiones, en concordancia con estudios previos. Aun cuando se ha mostrado que las personas con obesidad pueden llegar a tener la misma cantidad de fuerza que las personas con peso normal para pararse una vez estando sentados, la menor cantidad de repeticiones realizadas y menor velocidad en el flexo-extensión de rodillas por parte de los grupos sobrepeso/obesidad, reflejó que la capacidad funcional de las extremidades inferiores fue menor a los grupos normopeso ${ }^{30}$.

En la prueba de carrera de 12 metros, al contrario que en las dos pruebas anteriores, los resultados no mostraron diferencias entre género o estado nutricional. Aun cuando los niños con sobrepeso/obesidad fueron un 3,7\% más lentos que los niños de peso normal y las niñas con sobrepeso/ obesidad fueron un $3,1 \%$ más lentas que las niñas con peso normal. Se ha sugerido que los niños obesos tienen diferencias cinemáticas en la marcha, especialmente en la carrera, comparada con niños normopeso, mostrando menor candencia de pasos y velocidad ${ }^{30}$.

Es probable que en nuestro estudio la carrera de 12 metros no fuera una distancia suficientemente larga para detectar estas diferencias, de manera que los niños con IMC más alto pudieron mantener velocidades similares a los niños con normopeso durante aquella distancia. Estudios realizados en carreras más largas si han encontrado diferencias por estado nutricional y por género. Por ejemplo, un estudio ( $n=216$ escolares; 8-11 años) mostró que los escolares que poseían niveles superiores de condición física 
en el course Navette y carrera de $4 \times 10$ m presentaban una mayor tendencia a un estatus de peso corporal normal ${ }^{31}$.

El estudio de la relación entre el estado nutricional y el rendimiento académico en la infancia es un área emergente y con divergencias. Liang J et al (2014) hicieron una revisión de artículos desde 1976 al 2013 en las principales bases de datos médicas con el objetivo de determinar la relación entre obesidad, conductas relacionadas con la obesidad y funciones neurocognitivas en niños y adolescentes. Encontraron 77 artículos que cumplieron los criterios de inclusión metodológicos. De los cuales, solo 4 asociaban negativamente la obesidad y el bajo rendimiento escolar (entre edades de 7 a 11 años). A modo de ejemplo, Castelli et $\mathrm{al}^{32}$, encontraron una asociación inversa entre IMC y puntajes en test estandarizados de matemática y lenguaje en una muestra de 259 niños y niñas (9,5 $\pm 0,7$ años), y hubo 3 estudios en donde no se encontró una relación (en niños de 6-12 años). Según los autores, los resultados no concluyentes pueden deberse a las diferentes metodologías empleadas entre los estudios, a los diferentes tipos de test administrados, a posibles factores de confusión no controlados (edad, género, etnias, conductas saludables y comorbilidades) y a diferencias en los puntajes de corte para determinar el estado nutricional ${ }^{32}$. En nuestra investigación, no se encontraron diferencias en el rendimiento académico entre grupos de diferente estado nutricional o género. Las medias de matemáticas y lenguaje fueron altas en todos los grupos (cercanos al 6,0 en una escala de 1,0 a 7,0) comparadas con las medias nacionales de centros educativos públicos ${ }^{5,6}$, por lo que encontrar diferencias debido a un efecto techo, era poco probable; además, no se controlaron comorbilidades.

Por otra parte, en Chile se ha comenzado a estudiar la relación entre los hábitos de salud y el rendimiento escolar. En un estudio con escolares de $5^{\circ}$ a $9^{\circ}$ grado $(n=1271$; de 10-15 años) se determinó que quienes realizaban más de 4 horas de AF sistemática tenían más del doble de posibilidades de estar dentro de los puntajes más altos de test estandarizados nacionales de matemática y lenguaje ( $\geq$ percentil 75 (OR: 2,$1 ; 95 \%$ IC: $1,3-3,3)^{33}$. En otro estudio, Correa-Burrow et $\mathrm{a}^{11}$ en escolares $(n=1073)$ con una media de 13.1 años determinaron que quienes comían snacks menos saludables tenían peor rendimiento en test estandarizados de matemática y lenguaje. En Chile, los snacks no saludables más populares son los productos de pastelería, chocolates y golosinas que poseen altos niveles de azúcar, grasas saturadas y carbohidratos. Estudios previos hechos en animales sugieren que este tipo de alimentos mejoran el humor, disminuyen el estrés y controlan los síntomas de ansiedad ${ }^{34}$. Este fenómeno fue descrito por Dallman et al ${ }^{35}$ como la auto-medicación con alimentos.

Los grupos sobrepeso/obesidad declararon tener un mayor consumo de comidas no saludables con respecto a los grupos normopeso. En particular, el grupo m-spo consumió más snacks no saludables que el grupo m-np, hecho que podría estar asociado a que las niñas del grupo $\mathrm{m}$-spo tuvieron menores puntajes en la escala de ansiedad infantil (SCAS) con respecto a las niñas con normopeso. Los puntajes totales y de las subescalas de todos los grupos, superaron los valores de referencia para la población infantil y adolescente $(22,81 \text { para hombres y } 27,51 \text { para mujeres })^{18}$ situación que no deja de ser preocupante, pensando en la corta edad de nuestra muestra. No se encontraron diferencias en los niveles de ansiedad entre niños y niñas, hecho que contrasta con la mayoría de la literatura ${ }^{36}$. Una explicación posible es que los mayores niveles de ansiedad de las niñas con respecto a los niños se han encontrado alrededor de la pubertad cuando existe mayor presión social y familiar por tener un peso normal y perderlo si se ha ganado ${ }^{37}$.

Los grupos con peso normal, principalmente las niñas presentaron niveles de ansiedad mayores a los grupos sobrepeso/obesidad, hecho que también es divergente con estudios en humanos que han encontrado una asociación entre obesidad y patrones de consumo no saludables con altos niveles de ansiedad ${ }^{38}$. Esto podría deberse a que las investigaciones que han detectado mayores niveles de ansiedad evaluaron mujeres adolescentes en tratamiento para bajar peso o adolescentes con diagnóstico de ansiedad generalizada o depresión mayor ${ }^{39}$. En la misma línea, la ansiedad generada en la obesidad infantil podría ser explicada, en parte por la insatisfacción con la imagen corporal, hecho que se acrecienta en la pubertad y no es tan marcado en edaes tan tempranas como las evaluadas en nuestro estudio ${ }^{40}$.

Hasta la fecha no se encontraron estudios nacionales para comparar nuestros resultados, siendo el primero que estudia la relación entre los niveles de ansiedad y el estado nutricional de escolares tan jóvenes. Entre las limitaciones del estudio se encuentra que, se hizo difícil comparar los resultados obtenidos debido a la escasez de estudios de similares características, principalmente las variables de salud mental en edades tan precoces.

Por otra parte, el diseño de investigación limitó la opción de obtener relaciones causales de las variables. El tamaño de la muestra y el tipo de muestreo no permitió extrapolar los resultados a la población de niños de la provincia del Biobío. Tampoco se evaluó y analizó el impacto que tienen los profesores y los padres de los niños en las variables evaluadas. Se recomiendan estudios más amplios y con un diseño metodológico que permita caracterizar más profundamente la población objetivo y determinar con claridad la asociación que existe entre el estado nutricional, el rendimiento académico y la salud mental en escolares chilenos. En conclusión, niños (as) presentan altos niveles de malnutrición por exceso. Quienes presentaron mayores niveles de IMC/edad tenían peor condición física, un mayor perímetro de cintura, dedicaban menos horas semanales a realizar AF, comían más alimentos no saludables, pero no presentaron menor rendimiento académico. En particular, se observó que las niñas del grupo m-spo presentaron menores niveles de ansiedad. En espera de futuros estudios, los resultados obtenidos podrían darnos luces del actual estado nutricional, de los hábitos de salud, de la condición 
física, académica y mental de las escolares de la provincia del Biobío, Chile.

\section{RESUMEN}

En los últimos años, la obesidad ha aumentado y ello se ha asociado a una disminución de la condición física, problemas psicológicos y un bajo rendimiento escolar. El objetivo fue describir el estado nutricional, la condición física, el rendimiento escolar, el nivel de ansiedad y los hábitos de salud en escolares de enseñanza básica (6-7 años), y evaluar las diferencias entre sobrepesos/obesos y normo-pesos. Se realizaron dos estudios $(n=276)$ en escolares de $1^{\circ}$ y $2^{\circ}$ año básico. En ambos estudios, se obtuvieron parámetros antropométricos, de condición física y de rendimiento académico. Adicionalmente, en el $1^{\circ}$ estudio se midió el perímetro de cintura (PC) y en el $2^{\circ}$ hábitos de salud y sintomatología ansiosa. Niños y niñas presentaron altos niveles de sobrepeso y obesidad. Quienes poseían mayores niveles de IMC/edad tenían peor condición física, dedicaron menos horas semanales a actividad física sistemática y comían más alimentos no saludables. No hubo diferencias en rendimiento académico. Las niñas con sobrepeso/obesidad presentaron menores niveles de ansiedad que las con normopeso. Si bien estos resultados revelan el actual estado nutricional, condición física, académica y mental de una muestra de escolares de enseñanza básica, se necesitan más estudios para confirmar el alto grado de obesidad y sobrepeso detectado.

Palabras clave: Estado nutricional, educación básica, rendimiento escolar, conductas saludables, aptitud física, salud mental

\section{BIBLIOGRAFÍA}

1. Burrows R, Díaz E, Sciaraffia V, Gattas V, Montoya A, Lera L. Dietary intake and physical activity in school age children. Rev Med Chile 2008; 136: 53-63.

2. Crovetto M, Uauy R. Changes in household food and nutrient consumption in Metropolitan Santiago 1988-97 by income. Rev Med. Chile 2010; 138: 1091-1108.

3. Ministry of Health of Chile. Food consumption survey in Chile (ENCA). Santiago, Chile. 2014. [Available in http://web.minsal. cl/sites/default/files/ENCA-INFORME_FINAL.pdf], [Sought: January 22, 2015].

4. Ministry of Health of Chile, PUC, UAU. National Health Survey, 2009-2010. Santiago, Chile. [Available in: http://web. minsal.cl/portal/url/item/bcb03d7bc28b64dfe040010165012d23. pdf] [Sought: January 22, 2015].

5. World Health Organization (WHO), Global Recommendations on Physical Activity for Health. Ginebra, Suiza. [Available in: http://apps.who.int/iris/bitstream/10665/44441/1/9789243599977_ spa.pdf] [Sought: June 6, 2017].

6. Organization for Economic Co-operation and Development (OECD), Overweight and obesity among children, in Health at a Glance 2015: OECD Indicators, OECD Publishing, Paris. Francia. [Disponible en: [Available in: http://www.oecd-ilibrary. org/social-issues-migration-health/health-at-a-glance-2015_ health_glance-2015-en] [Sought: February 2, 2016].

7. Atalah E. Epidemiology of obesity in chile. Rev Med Clin Condes 2012; 23(2): 117-123.
8. Venn AJ. Overweight and obesity from childhood to adulthood: a follow-up of participants in the 1985 Australian schools health and fitness survey. Med J Aust 2007; 186: 458-460.

9. Centers for disease control and prevention (CDC). Overweight and obesity. [Available in: http://www.cdc.gov/obesity/index. html] [Sought: February 2, 2016].

10. Zametkin AJ, Zoon CK, Klein HW, Munson S. Psychiatric aspect of child and adolescents obesity: a review of the past 10 years. I Am Acad Child Adolesc Psychiatry 2004; 43(2): 134-150.

11. Datar A, Sturnm R, Magnabosco J. Childood overweight and academic performance: national study of kindergarteners and first-grades. Obes Res 2004; 12: 58-68.

12. Correa-Burrow $P$, Burrows $R$, Orellana $Y$, Ivanovic $D$. The relation between a healthy snack at school and academic outcomes: A population study in Chilean scholarchildren. Public Health Nutr 2015; 18(11): 2022-2030.

13. Ministry of Health of Chile, Technical norms for the supervision of children from 0 to 9 years in primary health care. National Child Health Program. 2014. Santiago, Chile. [Available in: http://web.minsal.cl/salud-infantil/] [Sought: February 2, 2016].

14. Castro-Piñero J, Artero EG, España-Romero V, Ortega FB,Sjöström $M$, Ruiz JR. Criterion-related validity of field-based fitness test in young: a sistematic review. Br J Sport Med 2010; 44(13): 934-943.

15. Ministry of Health of Chile, Healthy life prevention program for NCDs - Primary Care: Guidelines for Healthy Living. 2015. Santiago, Chile. [Available in: https://es.slideshare.net/ drjoseluiscontreras/orientacion-programa-vida-sana-2015-final] [Sought: February 2, 2016].

16. Castro-Piñero J Ortega FB, Artero EG. Girela Rejón MJ, Mora J, Sjöström $M$ et al. Assessing muscular streght in youth: usefulness of standing long jump as a general index of muscular fitness. J Strenght Cond Res 2010; 24(7); 1810-1817.

17. Monsalves-Alvarez M, Castro-Sepúlveda M, Zapata-Lamana $R$, Rosales-Soto G, Salazar Rodriguez G. Motor skills and nutritional status outcomes from a physical activity intervention in short breaks on preschool children conducted by their educators: a pilot study. Nutr Hosp. 2015;32(4):1576-1581.

18. Godard CM, Rodriguez M, Díaz N, Lera L, Salazar G, Burrow R. Value of a clinical test for assessing physical activity in children. Rev Med Chile 2008; 136: 1155-1162.

19. Godoy A, Gavino A, Carrillo F, Cobos MP, Quintero C. Factor structure of the Spanish version of the Spence Children Anxiety Scale (SCAS). Psicothema 2011; Vol 23(2): 289-294.

20. Whiteside SP, Brown AM. Exploring the utility of Spence children's anxiety scales parent-and chil-report forms in a North American sample. J Anxiety Disord 2008; 22: 14401446.

21. Espinoza-Silva M, Aguilar-Farias N. Nutritional status and physical capacity in 4 to 7-year-old students in a chilean public school 2014. Nutr Hosp 2015; 32(1): 69-74.

22. Szera G, Kovalskysa I, deGregorio MJ. Prevalence of overweight and obesity, and their relation to high blood pressure and central adiposity in students. Arch Argent Pediatr 2010: 108 (6): 492-498.

23. Masuet-Aumatell C, Ramon-Torrell JM, Banqué-Navarro $M$, Dávalos-Gamboa M, Montaño-Rodriguez SL. Prevalence of overweight and obesity in children and adolescents from Cochabamba (Bolivia); a cross-sectional study. Nutr Hosp 2013; 28(6): 1884-1891.

24. Roland-Cachera M, Deheeger M, Maillot M, Bellisle F. Early adiposity rebound: causes and consequences for obesity in 
child and adults. Int Obes 2006; S4; S11-17.

25. Koyama S. Ichikawa G, Kojima M, Shimura N, Sairenchi T et al. Adiposity reound and the development of metabolic syndrome. Pediatric 2014; 133(1): e114-119.

26. Ministry of Health of Chile. Diagnosis of the nutritional status of children under 6 years of age, pregnant women, nursing mothers and older adults, under control in the public health system. Santiago, Chile. 2013. [Available in: http://web.minsal.cl/ sites/default/files/DIAGNOSTICO_ESTADO_NUTRICIONAL_ DICIEMBRE_2013.pdf] [Sought: February 2, 2016].

27. Ortega FB, Ruiz JR, Castillo MJ. Physical activity, physical fitness, and overweight in children and adolescents: evidence from epidemiologic studies. Endocrinol Nutr 2013; 60(8): 458-469.

28. Delgado P, Caamaño F, Cresp M, Osorio A, Cofre A. Nutritional condition of schoolchildren and their association with levels of fitness and cardiovascular risk factors. Nutr Hosp 2015; 32(3): 1036-1041.

29. Castro-Piñero J, González-Montecinos IL, Mora J, Keating $X D$, Girela-Rejón MJ, Sjöström M et al. Percentile values for muscular strenght field test in children aged 6 to 17 years: Influence of weight status. J Strenght Cond Res 2009; 23(8): 2295-2310.

30. Pataky Z, Armand S, Müller-Pinget S, Golay A, Allet L. Effects of obesity on functional capacity. Obesity 2014; 22(1): 56-62.

31. Gálvez A, Rodríguez PL, Rosa A, Garcia-Cantó E, Perez J/, Tárraga $M L$, et al. Physical fitness level and its relationship with body weight status in school children. Nutr Hosp 2015; 31(1): 393-400.

32. Liang J, Matheson B, Kaye W, Boutelle K. Neurocognitive correlates of obesity and obesity-related behavoirs in children and adolescents. Int J Obes 2014; 38(4): 494-506.

33. Burrows $R$, Correa-Burrow $P$, Orellana $Y$, Almagia A, Lizana $P$, Ivanovic D. Scheduled pshysical activity is associated with better academic performance in Chilean school-age children. J Phys Act Health 2014; 11(8): 1600-1606.

34. Lalanza JF, Caimari A, del Bas JM, Torregrosa D, Cigarroa I, Pallàs $M$, et al. Effects of a post-weaning cafeteria diet in young rats: metabolic syndrome, reduced activity and low anxiety-like behaviour. PLoS One 2014; 9(1): e85049.

35. Dallman MF, Pecoraro NC, la Fleur SE. Chronic stress and comfort foods: self-medication and abdominal obesity. Brain Behav Inmun 2005; 19: 275-280.

36. Zhao J, Xing X, Wang M. Pyschometric properties of the Spence Children's Anxiety Scale (SCAS) in Mainland Chinese children and adolescents. J Anxiety Disord 2012; 26: 728-736.

37. Eresmis S, Cetin N, Amar M, Bukusoglu N, Akdeniz F, Goksen $D$. Is obesity a risk for psychopathology among adolescents? Pediatr Int 2004; 46: 296-301.

38. Burke NL, Storch EA. A meta-analysis of overweight status and anxiety in children and adolescents. J Dev Behav Pediatr 2015; 36(3): 133-145.

39. McElroy SL, Kotwal R, Malhotra S, Nelson EB, Keck PE, Nemeroff $C B$. Are mood disorders and obesity related? A review for the mental health professional. J Clin Psychiatry 2004; 65: 634-651.

40. Galvéz A, Rodríguez P, Guillamón A, García-Cantó E, PérezSoto J, Tarraga L, et al. Relationship between body weight status and self-concept in schoolchildren. Nutr Hosp 2015; 31(2): 730-736. 